\title{
THz Commensurate Echoes: Periodic Rephasing of Molecular Transitions in Free-Induction Decay
}

\author{
H. Harde, ${ }^{(a)}$ Søren Keiding, ${ }^{(b)}$ and D. Grischkowsky \\ IBM Watson Research Center, P.O. Box 218, Yorktown Heights, New York 10598
}

(Received 20 September 1990)

\begin{abstract}
We report the first study of coherent transients excited by ultrafast pulses of $\mathrm{THz}$ radiation. Using a newly developed optoelectronic source of well-collimated beams of subpicosecond pulses of $\mathrm{THz}$ radiation to excite $\mathrm{N}_{2} \mathrm{O}$ vapor, we have observed the subsequent emission from the vapor of coherent $\mathrm{THz}$ pulse trains extending to as long as $1 \mathrm{nsec}$. The origin of these subpicosecond $\mathrm{THz}$ pulses (echoes) is a periodic rephasing, during the free-induction decay, of the more than fifty coherently excited rotational lines with commensurate transition frequencies. From the decay and reshaping of the echoes the coherent relaxation time $T_{2}$ and the anharmonicity factor for the $\mathrm{N}_{2} \mathrm{O}$ molecule are evaluated.
\end{abstract}

PACS numbers: $42.50 . \mathrm{Md}, 42.50 . \mathrm{Kb}, 42.50 . \mathrm{Qg}$

Coherent transients resulting from the interaction of electromagnetic radiation with resonant systems were first studied for nuclear and paramagnetic spin systems at radio and microwave frequencies. ${ }^{1}$ Analogous studies in the optical regime were initiated with the photon-echo experiment, ${ }^{2}$ and since that time there have been extensive studies of coherent effects in both the visible and infrared regimes. ${ }^{3-5}$ While many of these phenomena are analogs of effects first observed in spin resonance, some of them do not have their spin-resonance counterpart because of propagation effects.

In this paper we report the first experimental and theoretical study of coherent transients excited by pulses of $\mathrm{THz}$ radiation. This newly accessible frequency range is important due both to the samples that can be investigated and to the unusual experimental conditions encountered with subpicosecond pulses of $\mathrm{THz}$ radiation. Commercially available submillimeter-wave microwave sources can only be modulated up to 0.2 nsec and can at most be tuned by $10 \%$ of their center frequency. In comparison the $\mathrm{THz}$ pulses used here are unique in terms of their pulse duration and corresponding bandwidth. They essentially consist of a single oscillation and are characterized by a transform-limited white spectrum in the $\mathrm{THz}$ range. This allows entire manifolds of resonance lines to be excited in the impact approximation. However, in contrast to ordinary absorption spectroscopy where only the spectral amplitudes are measured, and compared to infrared and optical investigations, where coherent transients are only detected as pulse intensities or as beat notes on a carrier, ${ }^{4-6}$ our experiments directly measure the actual electric field of the transmitted signals. We thereby determine both the spectral amplitudes as well as the phase of the transmitted components. Compared to coherence effects for nuclear- and electron-spin systems, ' the work reported here involves samples many wavelengths in length, so that in addition to the point response, propagation effects are also important. Because the subpicosecond pulses cannot be represented by a carrier frequency and a time-dependent pulse envelope, one cannot use the slowly varying envelope ap- proximation of coherent optics, ${ }^{3}$ and the concept of pulse area does not apply.

We have investigated coherent $\mathrm{THz}$ transients from $\mathrm{N}_{2} \mathrm{O}$ vapor. The propagation of a subpicosecond $\mathrm{THz}$ pulse through such a molecular vapor excites a multitude of rotational transitions in the impact approximation and causes the molecules to reradiate a free-induction-decay (FID) signal. ${ }^{1,3}$ Because the $\mathrm{N}_{2} \mathrm{O}$ molecule has an almost constant frequency spacing between the rotational lines, a periodic rephasing and dephasing of the entire ensemble of the more than fifty excited transitions is observed. This situation contrasts to that of water vapor with a multitude of incommensurate resonance lines, studied earlier by $\mathrm{THz}$ time-domain spectroscopy. ${ }^{7}$ Consequently, after the initial excitation pulse, the $\mathrm{N}_{2} \mathrm{O}$-vapor sample emits a series of uniformly spaced (39.8 psec) subpicosecond $\mathrm{THz}$ pulses (for a related phenomenon of infrared pulses emitted after excitation see Refs. 8 and 9). The rephasing process has a close relationship to that of spin and photon echoes. Because of this and because the frequencies are numerical multiples of the fundamental frequency, equal to the spacing between adjacent rotational lines, we term these periodic pulses "THz commensurate echoes." Because of the experimental system's exceptionally high signal-to-noise ratio, we have measured, with a time resolution of better than 0.5 psec, trains of echoes extending to beyond 600 psec. These echoes decay with the coherent relaxation time $T_{2}$, while Doppler dephasing can be completely neglected. From these observations $T_{2}$ can be directly determined. As will be described later in the paper, $T_{2}$ can still be measured when the absorption lines are so strongly overlapping that the rotational line structure is no longer observed in the frequency domain. From the echo repetition rate the frequency separation between the rotational lines is found, and because of the exceptionally high time resolution of the measurements, the anharmonicity in the line spacing can be detected as reshaping of the individual pulses.

The setup used to generate and detect the short pulses of $\mathrm{THz}$ radiation is depicted in Fig. 1 (a) and has been 

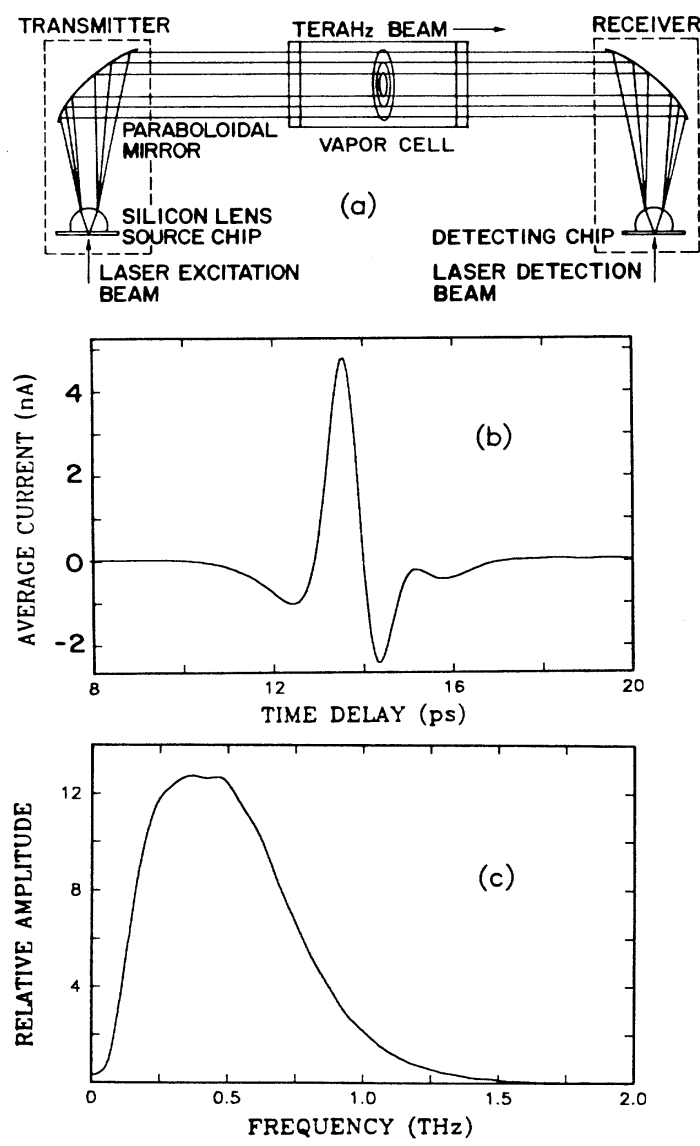

FIG. 1. Optoelectronic THz beam system. (a) THz optics, (b) measured electromagnetic pulse, and (c) amplitude spectrum of the pulse.

described earlier. ${ }^{7,10}$ A transmitting antenna with the bow-tie geometry and a receiving antenna consisting of a micron-sized dipole antenna imbedded in a coplanar transmission line were both optoelectronically driven by 70 -fsec pulses from a colliding-pulse mode-locked dye laser. The generated pulses of $\mathrm{THz}$ radiation are collimated by a silicon lens attached to the transmitting chip and directed onto a paraboloidal mirror that recollimates the radiation into a beam directed towards the receiver, where it is focused onto the receiving antenna. The time-dependent voltage induced across the receiving antenna is determined by measuring the collected charge (current) versus the time delay between the laser excitation pulses and the laser detection (gating) pulses. Such a measured transmitted $\mathrm{THz}$ pulse is shown in Fig. 1(b). The amplitude spectrum presented in Fig. 1(c) is a numerical Fourier transform of the measured pulse.

The measurements were performed on $\mathrm{N}_{2} \mathrm{O}$ vapor within a $38.7-\mathrm{cm}$-long cell having $2-\mathrm{cm}$-thick, 5 -cm-diam windows of $10-\mathrm{k} \Omega \mathrm{cm}$ silicon. ${ }^{10}$ For an evacuated cell we obtained the output pulse shown in Fig. 2(a). Here, in addition to the main pulse we see reflections from the

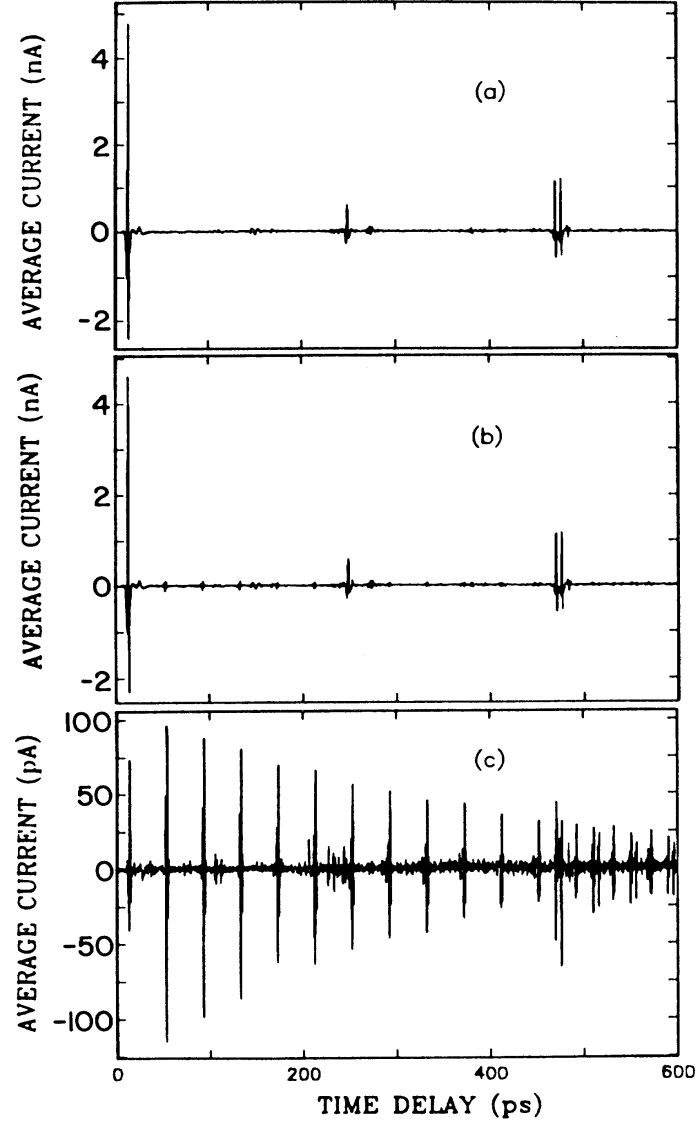

FIG. 2. (a) Measured transmitted $\mathrm{THz}$ pulse without $\mathrm{N}_{2} \mathrm{O}$ in the cell, (b) measured $\mathrm{THz}$ pulse with $120 \mathrm{hPa} \mathrm{N}_{2} \mathrm{O}$, and (c) free-induction-decay signal with the $\mathrm{THz}$ commensurate echoes given as the difference between (b) and (a).

transmitting antenna ( $250 \mathrm{psec})$ and two reflections from the windows $(470 \mathrm{psec})$. The other low-level structure is mainly due to the residual water vapor. ${ }^{7}$ When the cell is filled with $120 \mathrm{hPa}$ of $\mathrm{N}_{2} \mathrm{O}$ vapor, the output changes to that shown in Fig. 2(b), where the echo pulse train emitted by the vapor is just observable. The commensurate echoes are seen more clearly by plotting the difference, shown in Fig. 2(c), between the measurement [Fig. 2(b)] and the reference scan [Fig. 2(a)].

A closer look at the individual echoes of the train shows a significant reshaping with time, as illustrated by comparing the first, seventh, and fourteenth echoes emitted by the sample at 40,280 , and 560 psec after the initial excitation pulse [see Figs. 3(a)-3(c)]. This reshaping is caused by small deviations from the rigid-rotator model of the $\mathrm{N}_{2} \mathrm{O}$ molecules. As centrifugal forces can increase the moment of inertia of a rotating molecule, the frequency spacing between adjacent rotational lines is not constant but decreases slightly with increasing rotational quantum number $J$. This anharmonicity causes a gradual dephasing of the individual transitions and manifests itself as a change in the pulse shape. In analo- 

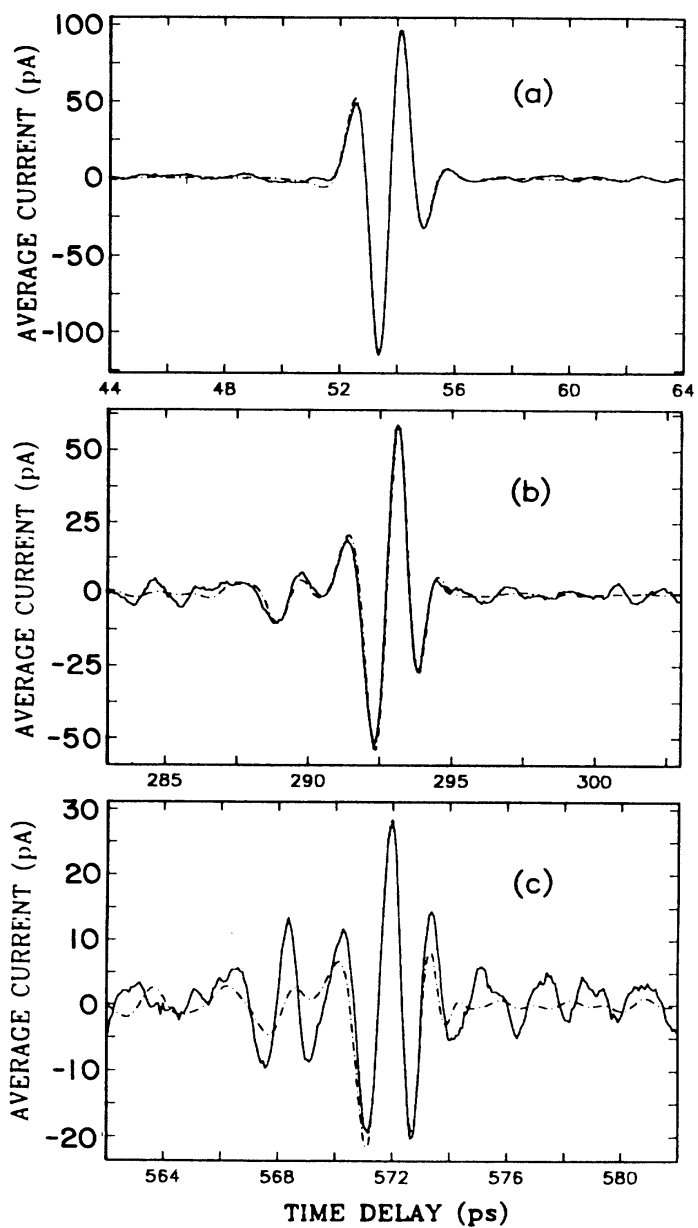

FIG. 3. Measurement of Fig. 2(c) (solid line) and calculation (dash-dotted line) of the pulse shape of (a) the first, (b) the seventh, and (c) the fourteenth $\mathrm{THz}$ commensurate echo on an expanded time scale.

gy to Doppler dephasing, where there is an induced polarization in the gas and by this the FID is washed out due to frequency shifts resulting from the motion of the molecules, we designate this phenomenon as centrifugal dephasing. This is the first time that this type of dephasing has been observed in the time domain.

Because we are in the low-intensity limit of the Maxwell-Bloch equations, their solution reduces to that of linear dispersion theory. ${ }^{3}$ We can then describe the interaction of the electric field with the sample in the frequency domain by introducing the absorption $\alpha(\omega)$ and dispersion $\Delta k$. The propagation of the pulse $E(z, t)$ through the sample can then be calculated from the well-known relationship

$E(z, t)=\int_{-\infty}^{\infty} E(\omega) e^{-i\left(\omega t-k_{0} z\right)} e^{i \Delta k(\omega)_{z}} e^{-\alpha(\omega)_{z} / 2} d \omega$.

The absorption coefficient for a single rotational transition of $\mathrm{N}_{2} \mathrm{O}$ from $J \rightarrow J+1$ assuming unpolarized radiation and or unoriented molecules can be written as

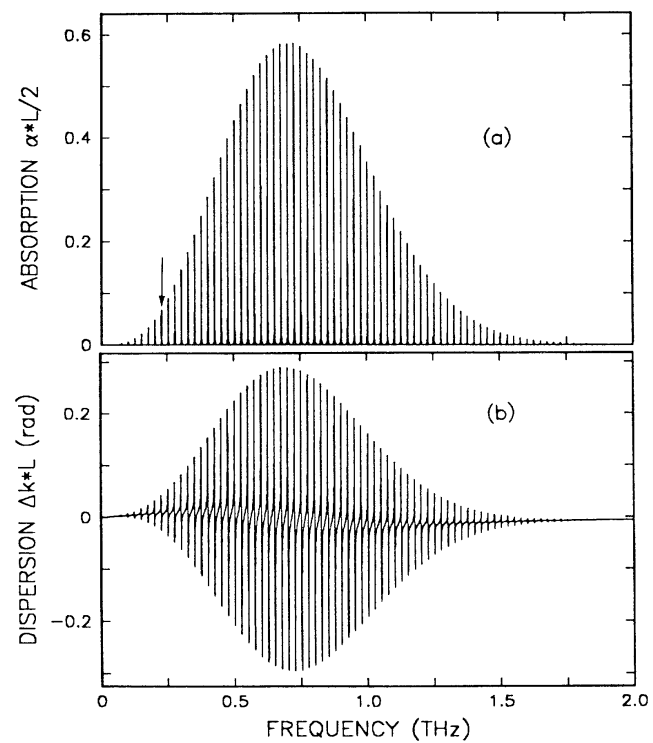

FIG. 4. (a) Calculated absorption and (b) dispersion of cell filled with $120 \mathrm{hPa} \mathrm{N}_{2} \mathrm{O}$.

$$
\begin{aligned}
& \alpha_{J}(\omega)=a_{J}(\omega) G_{A}\left(\omega, \omega_{J}\right) \text { with } \\
& \quad a_{J}(\omega)=\frac{p f_{0} \mu^{2} h B_{V} \omega^{2}}{6 n c \varepsilon_{0}(k T)^{3}}(J+1) e^{-h B_{V} J(J+1) / k T},
\end{aligned}
$$

where $B_{V}$ denotes the rotational constant of the vibrational state, $p$ the gas pressure, $f_{0}$ the fraction of molecules in the lowest vibrational state, $\mu$ the electric dipole moment, $h$ Planck's constant, $k T$ the thermal energy, and $G_{A}\left(\omega, \omega_{J}\right) / \pi$ a Van Vleck-Weisskopf absorption line shape, as given by the function in brackets of Eq. 13-16 of Ref. 11. The transition frequency is determined by $\omega_{J} / 2 \pi=2 B_{V}(J+1)-4 D_{V}(J+1)^{3}$, where $D_{V}$ is the centrifugal stretching constant. ${ }^{12}$ In a similar fashion $\Delta k_{J}(\omega)$ is given by

$$
\Delta k_{J}(\omega)=2 a_{J}(\omega) G_{N}\left(\omega, \omega_{J}\right) \omega_{J}^{2} / \omega\left(\omega_{J}^{2}-\omega^{2}\right),
$$

where $G_{N}\left(\omega, \omega_{J}\right)$ describes a Van Vleck-Weisskopf dispersion line shape, identical to the function in curly brackets in Eq. 13-15 of Ref. 11. The absorption $\alpha(\omega)$ and dispersion $\Delta k(\omega)$ are found by summing $\alpha_{J}(\omega)$ and $\Delta k_{J}(\omega)$ over all the transitions within the spectral width of the $\mathrm{THz}$ pulse.

The amplitude absorption $\alpha L / 2$ and the phase change $\Delta k L$ calculated for a pressure of $p=120 \mathrm{hPa}$ and a cell length of $L=0.387 \mathrm{~m}$ are shown in Fig. 4 using the constants $B_{V}=12.56163 \mathrm{GHz}, D_{V}=5.279 \mathrm{kHz}, \mu=0.166$ $\times 3.33 \times 10^{-30} \mathrm{~A} \mathrm{~s} \mathrm{~m}$, and $f_{0}=0.89$ for room-temperature $\mathrm{N}_{2} \mathrm{O}$ molecules. The only free parameter is the (FWHM) angular-frequency linewidth $\Delta \omega_{J}$, here assumed to be identical for all transitions with $\Delta \omega$ $=2 / T_{2}=2 \pi \times 670 \mathrm{MHz}$. With this spectral response the propagation of the $\mathrm{THz}$ beam through the $\mathrm{N}_{2} \mathrm{O}$ vapor can be calculated from Eq. (1). The theoretical results 


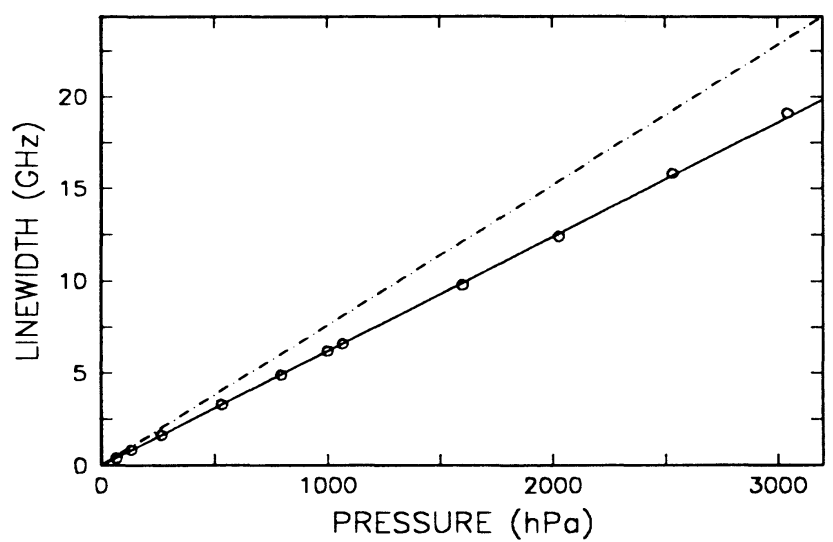

FIG. 5. Self-pressure broadening of $\mathrm{N}_{2} \mathrm{O}$ vs vapor pressure for the transition $J=8 \rightarrow 9$ at $226.1 \mathrm{GHz}$.

are in excellent agreement with the measurements in pulse shape and absolute amplitude, for the exciting pulse as well as the coherently generated pulse train. This is demonstrated by the comparison in Fig. 3 between theory and experiment for the first, seventh, and fourteenth echoes of the FID.

From molecular collision theories ${ }^{13,14}$ and experimental studies of rovibronic lines in the $v_{3}$ band ${ }^{15}$ it is known that the linewidth $\Delta \omega_{J}$ decreases slightly with increasing $J$. In order to compare our results with frequency-domain experiments measuring individual lines, we used a single transition as a reference line, and assumed the same linewidth variation as found in Ref. 15 to calculate the entire absorption and dispersion spectra. A fit of the simulated pulse train to the measurement then gives the pressure broadening shown in Fig. 5 for the transition $J=8 \rightarrow 9$ at $226.1 \mathrm{GHz}$ indicated by the arrow in Fig. 4 . The solid line interpolates between the measurements and demonstrates a linear dependence of the linewidth over a pressure range of almost 2 orders of magnitude. At the higher pressures the broadening has become as large as the line spacing, and adjacent transitions strongly overlap. An accurate analysis of such data in the frequency domain would be quite difficult. The slope of the solid line gives the self-pressure broadening parameter for the $J=8 \rightarrow 9$ transition at room temperature (for the temperature correction see Ref. 16) of $C_{W}=6.20 \mathrm{MHz} /$ $\mathrm{hPa}=8.26 \mathrm{MHz} /$ Torr (related to the FWHM) in excellent agreement with the infrared measurements, ${ }^{15}$ but roughly $10 \%$ smaller than the value obtained from microwave experiments. ${ }^{16}$ If propagation effects were neglected in the analysis, significantly larger linewidths would be obtained as indicated by the dash-dotted line in Fig. 5. This discrepancy is due to absorption, which under our experimental conditions cannot be assumed to be small compared to unity (see Fig. 4). At the line center saturation effects cause an additional spectral broadening manifest in the time domain as a faster decay. A reshaping of the propagating $\mathrm{THz}$ pulses and commensurate echoes due to the dispersion is not observed under our experimental conditions, but has to be considered for longer propagation lengths.

At much higher pressures than used in our experiments, the rotational lines overlap so severely that the entire spectral envelope appears as a smooth curve similar to a broad single resonance line. An analysis of the pressure-broadening data in the frequency domain then becomes impossible. However, at these high pressures weak echoes are still expected. For example, for a pressure of $10000 \mathrm{hPa}$ for which $T_{2}=5.2 \mathrm{psec}$, we calculate an amplitude for the first commensurate echo of approximately $\frac{1}{1000}$ of that of the excitation pulse. Given our limiting signal-to-noise ratio of $10000: 1$, this echo should be observable. Presently, our inability to completely eliminate the water vapor from our experimental apparatus precludes the observation. However, the calculated result illustrates the power of the $\mathrm{THz}$ commensurate echoes to measure $T_{2}$ in the situation of strongly overlapping lines.

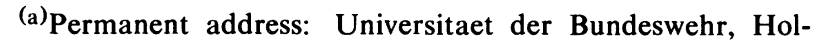
stenhofweg 85, 2 Hamburg 70, Federal Republic of Germany.

(b) Permanent address: Institute of Physics, University of Odense, DK-5230 Odense M, Denmark.

${ }^{1}$ A. Abragam, The Principles of Nuclear Magnetism (Oxford Univ. Press, New York, 1961).

${ }^{2}$ N. A. Kurnit, I. D. Abella, and S. R. Hartmann, Phys. Rev. Lett. 13, 567 (1964).

${ }^{3}$ L. Allen and J. H. Eberly, Optical Resonance and TwoLevel-Atoms (Wiley, New York, 1975).

${ }^{4}$ R. G. Brewer, in Frontiers in Laser Spectroscopy, edited by R. Balian, S. Haroche, and S. Liberman (North-Holland, Amsterdam, 1977), p. 341.

${ }^{5}$ R. L. Shoemaker, in Laser and Coherence Spectroscopy, edited by J. I. Steinfeld (Plenum, New York, 1978), p. 197.

${ }^{6}$ M. Rosatzin, D. Suter, W. Lange, and J. Mlynek, J. Opt. Soc. Am. B 7, 1231 (1990).

${ }^{7}$ M. van Exter, Ch. Fattinger, and D. Grischkowsky, Opt. Lett. 14, 1128 (1989).

${ }^{8}$ K. L. Foster, S. Stenholm, and R. G. Brewer, Phys. Rev. A 10, 2318 (1974).

${ }^{9}$ M. Woerner, A. Seilmeier, and W. Kaiser, Opt. Lett. 14, 636 (1989).

${ }^{10} \mathrm{M}$. van Exter and D. Grischkowsky, IEEE Trans. Microwave Theory Tech. 38, 1684 (1990).

${ }^{11}$ C. H. Townes and A. L. Schawlow, Microwave Spectroscopy (Dover, New York, 1975).

${ }^{12}$ A. G. Maki, J. S. Wells, and M. D. Vanek, J. Mol. Spectrosc. 138, 84 (1989).

${ }^{13}$ P. W. Anderson, Phys. Rev. 76, 647 (1949).

${ }^{14}$ D. Robert and J. Bonamy, J. Phys. (Paris) 40, 923 (1979).

${ }^{15}$ M. Margottin-Maclou, P. Dahoo, A. Henry, and L. Henry, J. Mol. Spectrosc. 111, 275 (1985).

16 J. M. Colmont and N. Semmoud-Monnanteuil, J. Mol. Spectrosc. 126, 240 (1987). 